\title{
Predissociation and autoionization of triplet Rydberg states in molecular hydrogen
}

\author{
Laura Dinu \\ FOM-Institute for Atomic and Molecular Physics (AMOLF), Kruislaan 407, 1098 SJ Amsterdam, \\ The Netherlands \\ Yan J. Picard \\ Laboratoire des Collisions Atomiques et Moleculaires Universite Paris-Sud, Batiment 35191405 Orsay \\ Cedex, France \\ Wim J. van der Zande \\ FOM- Institute for Atomic and Molecular Physics (AMOLF), Kruislaan 407, 1098 SJ Amsterdam, \\ The Netherlands and NSRIM, University of Nijmegen, Toernooiveld 1, 6525 ED Nijmegen, The Netherlands
}

(Received 25 March 2004; accepted 24 May 2004)

\begin{abstract}
We present single-photon spectroscopy in molecular hydrogen starting from the metastable $c^{3} \Pi_{u}^{-}$ state to a number of triplet $n d$-Rydberg states $(v=0-4, n=12-20)$. Using fast beam spectroscopy both the autoionization channel and the predissociation channel are quantified, field free, as well as with small electric fields. Coupling with the $i^{3} \Pi_{g}$ state is assumed to be responsible for field-free predissociation of the $v=0$ Rydberg levels. The stronger observed predissociation channel of the $v=1$ Rydberg levels is due to the nonadiabatic interaction with the $h^{3} \Sigma_{g}^{+}$state in combination with $l$ mixing due to an external electric field. No direct evidence is found for possible electric field induced predissociation of the gerade Rydberg states by low lying ungerade states. The competition between autoionization and predissociation is discussed in terms of possible consequences for dissociative recombination involving low energy electron collisions with the $\mathrm{H}_{2}^{+}$molecular ion.
\end{abstract}

(C) 2004 American Institute of Physics. [DOI: 10.1063/1.1773157]

\section{INTRODUCTION}

The spectroscopy of molecular hydrogen has been studied in great detail over many decades with many different techniques. Most of the molecular states in hydrogen are Rydberg states, which have one confined orbital and one more diffuse outer orbital. The lower lying states have been calculated with very high precision by Kołos and Wolniewicz. ${ }^{1-3}$ The Rydberg states have rich dynamics with radiation, autoionization, and predissociation as potential decay channels. Most of these processes have been studied not only experimentally but also theoretically, often employing multichannel quantum defect theory (MQDT). ${ }^{4,5}$ Most research has been directed to the $p$-electron Rydberg series, being accessible from the electronic ground state with one photon. In the ungerade Rydberg series, also higher angular momentum ungerade states have been studied ( $f$ series) via electron excitation from the ground state followed by twophoton excitation. ${ }^{6,7}$ Using multiphoton excitation also the $d$ and $s$-gerade Rydberg series have been detected and characterized. ${ }^{8}$ In a light molecule such as $\mathrm{H}_{2}$, the triplet Rydberg series are not accessible from the singlet electronic ground state. Still, these Rydberg states have been studied starting from the long-lived metastable $c^{3} \Pi_{u}^{-}$state, which has an excitation energy of $\approx 11.6 \mathrm{eV}$, and which may be produced by low energy electron impact or using chargetransfer collisions between molecular hydrogen ions and alkali atoms, such as sodium, rubidium, or cesium. ${ }^{9,10}$ For these reasons, most studies in triplet hydrogen have focussed on the $d$-gerade Rydberg series. ${ }^{11-13}$ To the best of our knowledge no triplet $n p$-ungerade Rydberg series have been detected. Using microwave spectroscopy, Lundeen and coworkers have measured high-resolution spectra of nonpenetrating high $l$ states. ${ }^{14,15}$ Hyperfine structure in low $l$ states of very high $n$-Rydberg states has been recently reported in a beautiful series of experiments. ${ }^{16}$

The electronic structure of molecular hydrogen results in dynamics, which differs strongly for different symmetries and multiplicities. Molecular hydrogen has doubly excited states in which both electrons occupy an excited orbital. The lowest doubly excited state is the $\left(2 p \sigma_{u}\right)^{2}{ }^{1} \Sigma_{g}^{+}$state. This state crosses the ionic ground state near the outer turning point of its second $\left(v^{+}=1\right)$ vibrational level (see Fig. 1). The lowest repulsive ungerade state and the lowest triplet gerade/ungerade states are the $\left(2 p \sigma_{u} 2 s \sigma_{g}\right)^{1,3} \Sigma_{u}^{+}$and $\left(2 p \sigma_{u} 2 p \pi_{u}\right)^{3} \Pi_{g}$ states, which cross the ionic ground state near the outer turning point of the $v^{+}=5-6$ vibrational levels. The different doubly excited characters have large effects on the lower lying adiabatic electronic states. The $\left(2 p \sigma_{u}\right)^{2}{ }^{1} \Sigma_{g}^{+}$character is responsible for the double well character in the first members of the $\left(n s \sigma_{g}\right){ }^{1} \Sigma_{g}^{+}$states. The $\left(2 p \sigma_{u} 2 p \pi_{u}\right)^{3} \Pi_{g}$ doubly excited state is visible in the potential barrier in the $(3 d \pi) i^{3} \Pi_{g}$ state. Also in the next member of the ${ }^{3} \Pi_{g}$ series, the $(4 d \pi)^{3} \Pi_{g}$ state, the effect of the doubly excited character is present. Vibrational autoionization of $(4 d \pi)^{3} \Pi_{g}$ levels excited from high $v=9-12$ of the $c^{3} \Pi_{u}^{-}$state, with a change of up to ten vibrational quanta has been observed ${ }^{17,18}$ and interpreted using MQDT. ${ }^{19}$ This behavior was attributed to the interaction of singly 


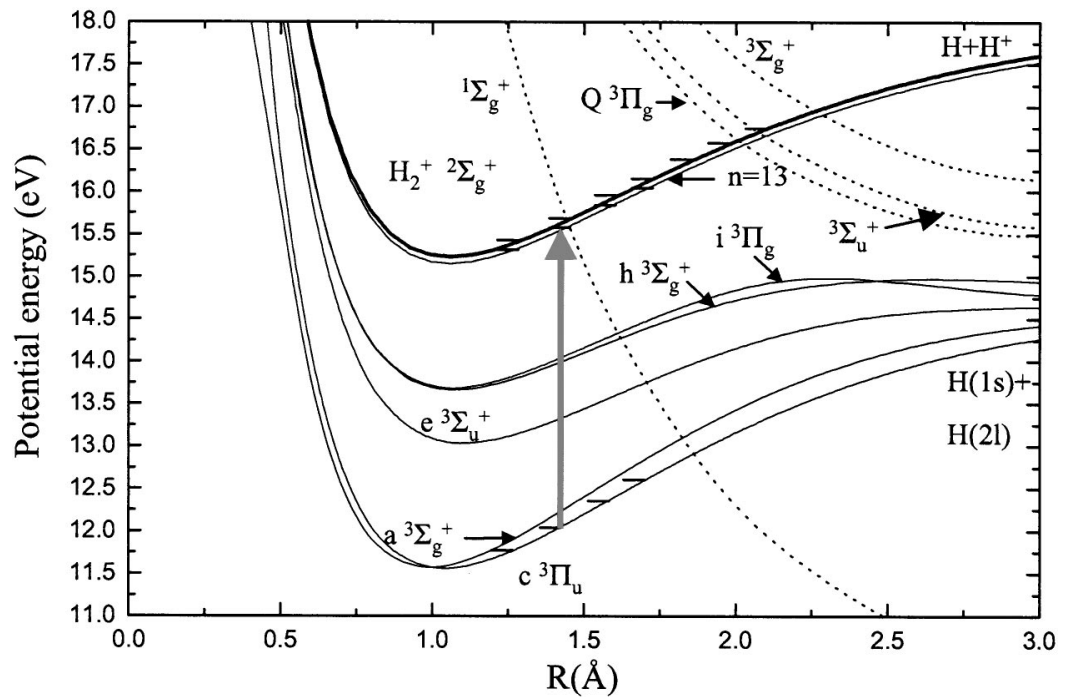

FIG. 1. Potential energy curves for molecular hydrogen. The black lines are the diabatic potentials for molecular hydrogen from Ref. 35. The repulsive curves are taken from Ref. 24. excited $(4 d \pi)^{3} \Pi_{g}$ state and the doubly excited $\left(2 p \sigma_{u} 2 p \pi_{u}\right) Q_{1}^{3} \Pi_{g}$ state.

Apart from the intrinsic interest in understanding and potentially manipulating the decay dynamics of the Rydberg states, a direct relation exists between the dynamic behavior of these Rydberg states and the efficiency and dynamics of low energy electron collisions on molecular hydrogen, $\mathrm{H}_{2}^{+}$ $+e^{-} \rightarrow \mathrm{H}(1 s)+\mathrm{H}(n l)+\mathrm{KER}$. This process is called dissociative recombination (DR). The kinetic energy of the fragments depends on the internal state of the molecular ion, the collision energy, and the internal energy of the excited $\mathrm{H}(n l)$ hydrogen fragment. In accordance with the concepts of the very successful multichannel quantum defect theory, low energy collisions of electrons with the molecular ion core have a large resemblance with the interactions between the Rydberg electron and the ionic core. The latter interactions cause the perturbations and decay of the associated Rydberg states. Real electron collisions sample all partial waves and both multiplicities. In many calculations involving $\mathrm{DR}$ of $\mathrm{H}_{2}^{+}$only the so-called direct DR process through the capture of an $\epsilon d \sigma_{g}$ electron in the doubly excited $\left(2 p \sigma_{u}\right)^{2}{ }^{1} \Sigma_{g}^{+}$-repulsive state is taken into account. ${ }^{20-23}$ The logical consequence of this restriction is that neither the triplet channels nor the ungerade partial waves are considered to result in DR. The DR rate at $300 \mathrm{~K}$ (electron temperature) is $2 \times 10^{-8} \mathrm{~cm}^{3} \mathrm{~s}^{-1}$, a value that is significantly smaller than the DR rate of many other species.

Next to the DR mechanism that involves capture of the continuum electron into a doubly excited state, an alternative mechanism has been postulated for the cases of $\mathrm{HeH}^{+}$and $\mathrm{H}_{2}^{+} \cdot{ }^{24,25}$ The observation of a significant DR rate of $\mathrm{HeH}^{+}$ required a new mechanism as this ion lacks a doubly excited state with a crossing through the bottom of the ionic ground state potential curve. In this mechanism, electron capture occurs directly into the dissociation continuum of lower lying Rydberg $(n=3)$ states in a quasi-one-electron process driven by the nuclear kinetic energy $d / d R$ operator. Logically speaking, this process may contribute to the DR rate in molecular hydrogen for the triplet channels, as well as for the singlet ungerade channel.
We present here single-photon spectroscopy in molecular hydrogen starting from the metastable $c^{3} \Pi_{u}^{-}$state to a number of triplet $n d$-Rydberg states $(v=0-4 ; n=12-16$ for $v=0)$. Using fast beam spectroscopy both the autoionization channel and the predissociation channel are quantified. We believe that an observable competition between autoionization and predissociation of these Rydberg states is a good indication or may be even a necessary (but not always sufficient) condition for DR to be efficient. By exciting the Rydberg states in a static electric field, these experiments reveal dynamics of both ungerade and gerade Rydberg states. These Rydberg states have been observed before by Eyler and Pipkin $^{6}$ and Knight and Wang. ${ }^{12}$ These authors detected the different levels using vibrational autoionization. Hence, the vibrational ground state levels remain undetected.

In the present experiment, the vibrational ground state is observed by detecting predissociation products with such detail that the initial state is rovibrationally resolved. The present experiment uses the same microchannel plate (MCP) detector to detect the products of autoionization $\left(\mathrm{H}_{2}^{+}\right.$ions $)$ and predissociation $\mathrm{H}-\mathrm{H}$ pairs), which makes it possible to assess the absolute branching.

In the following, we present our experimental setup (Sec. II), followed by a more detailed description of the triplet Rydberg levels in Hund's case $d$ in combination with the effect of applying a weak electric field (Sec. III). The experimental results are described in Sec. IV. Section V contains an interpretation of the results, including the consequences for the DR process.

\section{EXPERIMENT}

The experimental setup is shown in Fig. 2. The positive ions are produced in a hollow cathode discharge ion source and extracted at $5 \mathrm{kV}$. The ions of interest, $\mathrm{H}_{2}^{+}$, are mass selected by a Wien filter and introduced in a fast beam apparatus. By means of four deflection plates, the beam is guided through the apertures of the apparatus. Two Einzel lenses are used to focus the beam through the exit aperture of the ion source region and to reduce the radial size of the 


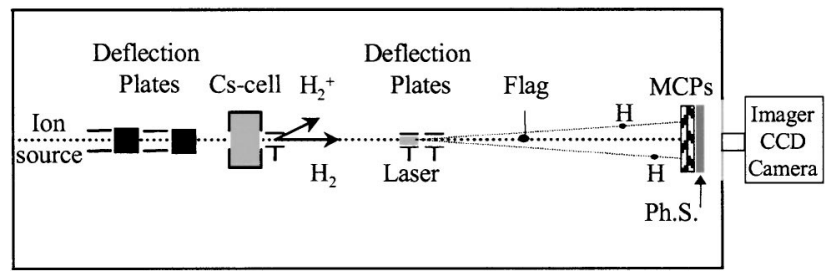

FIG. 2. Schematics of the apparatus. The molecular ion beam is produced in a hollow cathode ion source. The beam is extracted at $5 \mathrm{keV}$ and neutralized via charge-transfer reactions with Cs. A pulsed laser beam crosses at $90^{\circ}$ the neutral molecular beam. Once the foton is absorbed, predissociation and autoionization are the dominant decay paths of these high Rydberg states. A three-dimensional detector records the position $(x, y)$ and the arrival time $t$ of each detected particle.

beam in the region of interaction with the laser. After $\approx 170$ $\mathrm{cm}$ the beam passes through a cell of Cs vapor where chargeexchange processes take place. Part of the $\mathrm{H}_{2}^{+}$ion beam is neutralized and metastable excited states of molecular hydrogen are populated $\left[\mathrm{H}_{2}^{+}\left(X^{2} \Sigma_{g}^{+}\right)+\mathrm{Cs} \rightarrow \mathrm{H}_{2}\left(c^{3} \Pi_{u}^{-}\right)+\mathrm{Cs}^{+}\right]$. The residual ion beam is deflected after the collision cell. The neutral beam travels undisturbed along the setup axis and $50 \mathrm{~cm}$ away from the cell it passes a $1 \mathrm{~mm}$ diaphragm and enters the interaction region with the laser. The molecular beam and the laser cross at $90^{\circ}$. The laser system used for this experiment consists of a $50 \mathrm{~Hz}$ Spectra Physics Nd-YAG (yttrium-aluminum-garnet) laser pumping a Lambda Physik ScanMate dye laser. The output of the ScanMate (around 690 $\mathrm{nm}$ ) is frequency doubled by a KDP-C nonlinear crystal. The UV laser light has an energy of $5 \mathrm{~mJ}$ per pulse and it is line focused into the apparatus onto the molecular beam. The length of the line focus $(1-2 \mathrm{~cm})$ determines the overlap region between the molecular beam and the laser. Photons are absorbed and high Rydberg states are populated. The lifetime of these Rydberg states is short compared with the time of flight of the molecules in the laser region $(\sim 15 \mathrm{~ns} /$ $\mathrm{cm})$ therefore we can say that autoionization and predissociation occur inside the laser focus. The possible reactions are

$$
\begin{aligned}
\mathrm{H}_{2}+h \nu & \rightarrow \mathrm{H}_{2}^{*}(\text { Rydberg }) \rightarrow \mathrm{H}_{2}^{+}+e^{-} \\
& \rightarrow \mathrm{H}_{2}^{*}(\text { Rydberg }) \rightarrow \mathrm{H}+\mathrm{H}^{-} .
\end{aligned}
$$

If predissociation [see Eq. (2)] occurs, two hydrogen atoms fly apart with a kinetic energy corresponding to the internal state of the Rydberg molecule. After a time of flight of $\sim 1.8 \mu$ s the atoms hit the time- and position-sensitive detector. By measuring the kinetic energy release in the predissociation process, useful information can be extracted concerning the molecular Rydberg state (the energy of the state, the angular orientation of the molecule, etc.).

If the molecule is oriented perpendicular to the setup axis $z$ while dissociating, the kinetic energy released is encoded in the distance between the fragments. If the molecule is oriented along the $z$ axis, the fragments arrive in the same spot on the detector, but with a maximum time difference. For a randomly oriented molecule, the kinetic energy release can be expressed as

$$
\operatorname{KER}=\frac{E_{0}}{4 L^{2}}\left(D^{2}+v_{0}^{2} \Delta t^{2}\right),
$$

where $E_{0}$ is the kinetic energy of the beam $(5 \mathrm{keV}), L$ is the distance between laser region and detector $(125 \mathrm{~cm}), D$ is the distance between the fragments on the detector, $v_{0}$ is the velocity of the beam $\left(\sim 7 \times 10^{5} \mathrm{~m} / \mathrm{s}\right)$, and $\Delta t$ is the arrival time difference of the fragments.

In order to determine the kinetic energy release, both fragments must be detected. Two particles are recognized as fragments of the same molecule if their center of mass coincide with the center of mass of the ion beam.

The detector consists of a stack of two microchannel plates and a $P 46$ phosphor screen. A double exposure charge-coupled device (CCD) camera is placed behind the phosphor screen. The camera retrieves the $(x, y)$ coordinates of the spots with $80 \mu \mathrm{m}$ resolution and the arrival time of the particles with $1 \mathrm{~ns}$ resolution. ${ }^{26,27}$ The camera is the master clock of the experiment and it runs at $25 \mathrm{~Hz}$.

\section{RYDBERG STATES}

The Rydberg states that are studied in this paper may be described as a hydrogenic Rydberg electron orbiting a $\mathrm{H}_{2}^{+}\left({ }^{2} \Sigma_{g}^{+}\right)$ion core. We use Hund's case $d$ to describe these states. The angular momentum of the excited electron $\mathbf{l}$ is decoupled from the molecular axis and is coupled to the angular momentum of the ionic core $\mathbf{R}$. The total angular momentum $\mathbf{N}$ is the vector sum $\mathbf{N}=\mathbf{R}+\mathbf{l}$. The initial $c^{3} \Pi_{u}^{-}$ state is described in Hund's case $b$. In this coupling scheme the electronic orbital angular momentum $\mathbf{L}$ couples strongly to the molecular core and the projection $\Lambda$ is a good quantum number. The rotational states are indexed according to $N_{c}$, the total angular momentum exclusive of spin. We use the index $c$ to distinguish it from the total angular momentum of the Rydberg states, $N$. In the initial $c^{3} \Pi_{u}$ state only one of the parity states is metastable; the $c^{3} \Pi_{u}^{+}$state is rapidly predissociated by the $b^{3} \Sigma_{u}^{+}$state. As a consequence, the parahydrogen occupies the even angular momentum states, while the ortho-hydrogen occupies the odd angular momentum states. From the $c$ state, with a $2 p \pi_{u}$ outer electron, in the single-electron picture, either $n d$ or $n s$ states can be excited. The transition strength of $n s \leftarrow 2 p$ is smaller than the $n d$ $\leftarrow 2 p$ by a factor of $30 .{ }^{13}$ Hardly any $n s$ transitions have been reported so far. To the best of our knowledge the $4 s^{3} \Sigma_{g}^{+}$is the only Rydberg state (with $n$ larger than 3) that has been detected. ${ }^{28}$

The transition to the Rydberg state is characterized by $\mathrm{H}_{2}\left(c^{3} \Pi_{u}^{-}\right)+h \nu \rightarrow \mathrm{H}_{2}^{*}\left({ }^{2} \Sigma_{g}^{+}+n d\right)$. The energy levels of the initial $c^{3} \Pi_{u}^{-}$state are taken from Dieke's table. ${ }^{29}$ The values are corrected by $149.6 \mathrm{~cm}^{-1}$. 7,30 The constants for the $\mathrm{H}_{2}^{+}\left({ }^{2} \Sigma_{g}^{+}\right)$are $\omega_{e}=2321.7 \mathrm{~cm}^{-1}, \omega_{e} \chi_{e}=66.2 \mathrm{~cm}^{-1}, B_{e}$ $=30.2 \mathrm{~cm}^{-1}, \alpha_{e}=1.68 \mathrm{~cm}^{-1}$, and $D_{e}=0.618 \mathrm{~cm}^{-1} \cdot 31$

In order to estimate the energy of the rotational Rydberg states we used an $a b$ initio model described by Eyler and Pipkin. ${ }^{11}$ This model has been successfully applied. ${ }^{7,12}$ In brief, the lowest-order couplings that perturb the Rydberg electron from hydrogenic energies are considered. There are two effects: (1) The potential experienced by the outer elec- 
tron is expanded into a multipole series, in which the quadrupole moment of the $\mathrm{H}_{2}^{+}$core is the first nonzero term; (2) the presence of the outer electron induces mixing of the core states and this is taken into account by considering the polarizability of the $\mathrm{H}_{2}^{+}$core. The matrix elements of the polarizability and quadrupole moment perturbations are obtained in a case- $d$ basis. The derived first-order energy corrections are

$$
\begin{aligned}
& E^{(0)}=-A C Q(v, R)-\frac{1}{2} B \alpha(v, R)-\frac{1}{3} A B \gamma(v, R), \\
& A=\frac{3 Y(Y-1)-4 R(R+1) L(L+1)}{2(2 L-1)(2 R-1)(2 L+3)(2 R+3)}, \\
& B=\frac{\frac{1}{2}\left[3 n^{2}-L(L+1)\right]}{n^{5}\left(L+\frac{3}{2}\right)(L+1)\left(L+\frac{1}{2}\right) L\left(L-\frac{1}{2}\right)}, \\
& C=\frac{1}{n^{3}(L+1)\left(L+\frac{1}{2}\right) L}, \\
& Y=R(R+1)+L(L+1)-N(N+1) .
\end{aligned}
$$

The $Q(v, R), \alpha(v, R)$, and $\gamma(v, R)$ are polarizability and quadrupole constants. Eyler and Pipkin calculated these constants for $v=0-3$ and $R=0-3$. They observe a weak dependence of these constants on the rotational quantum number and a stronger dependence on the vibrational level. ${ }^{11} \mathrm{We}$ note that Eq. (4) only accounts for the effect of the longrange forces felt by the Rydberg electron and does not take into account the penetration of the electron from the ionic core.

In our calculation, we use Eq. (4) and the values for the polarizability and quadrupole constants. For $R=4-6$ we use extrapolated values of the constants. The transitions $\mathrm{H}_{2}^{*}(v, R, N) \leftarrow \mathrm{H}_{2}\left(c^{3} \Pi_{u}^{-}\right)\left(v, N_{c}\right)$ are indexed by $\left(R, N, N_{c}\right)$. The allowed optical transitions are characterized by $\Delta N$ $=0, \pm 1$. The conservation of ortho- and para-hydrogen in the transition demands $R=N_{c}, N_{c} \pm 2$. The change of two units in rotational angular momentum is unlikely because of the atomiclike character of the excitation process characterized by a weak interaction of the Rydberg electron with the ionic core. These arguments result in an observed propensity for $R=N_{c}$. The shape of the potential curves and the similarity of the vibrational constants of the initial state $\left(\omega_{e}\right.$ $\left.=2466.9 \mathrm{~cm}^{-1}\right)$ and the ionic state $\left(\omega_{e}=2321.7 \mathrm{~cm}^{-1}\right)$ explains the prevailing of $\Delta v=0$ transitions over $\Delta v \neq 0$ transitions.

Autoionization processes involve an interaction of the outer electron with the ionic core, which results in a decrease of the vibrational energy with one quantum, accompanied by excitation of the Rydberg electron in the ionization continuum. Predissociation also involves the interaction of the outer electron with the ionic core and it conserves the overall parity and the total angular momentum of the states involved. The predissociation channels either have the same overall symmetry (homogenous interaction) or, for example, in case of rotational coupling, electronic orbital angular mo-
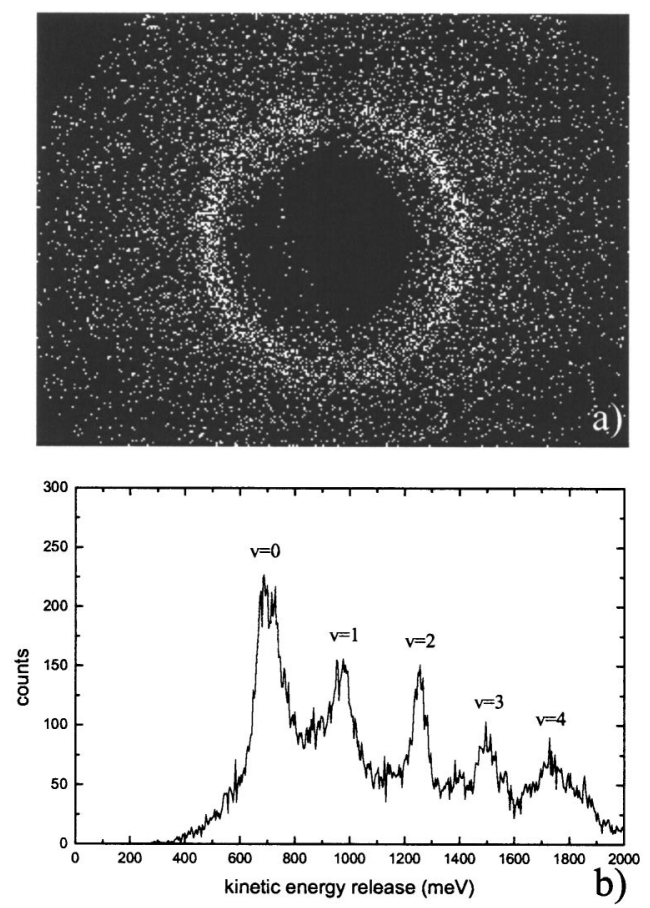

FIG. 3. (a) Raw data of the $n=13, v=0$ predissociation scan between 346.13 and $348.19 \mathrm{~nm}$ ( $G_{7}$ line); (b) KER histogram for a larger scan $343.3-348.3 \mathrm{~nm}$; five peaks are observed, indicating excitation from the $v=0-4$ levels in the $c^{3} \Pi_{u}^{-}$state.

mentum and rotational angular momentum are exchanged. In the present experiments, the excited Rydberg states dissociate into $\mathrm{H}(1 s)+\mathrm{H}(2 l)$ fragment pairs.

\section{RESULTS}

The experiments take place in a fast beam apparatus, which allows a large detection probability of the fragments on a MCP detector. The detection probability of ionic $\mathrm{H}_{2}^{+}$is similar to the detection probability of the atomic fragments $\mathrm{H}(1 s)$ and $\mathrm{H}(2 l)$, therefore comparison between autoionization and predissociation yields is possible. We note that the metastable hydrogen molecules are excited in a crossed beam setup. All these excited molecules decay and the fragments are detected. Hence, the signal strength does not reflect the decay rates but the excitation probabilities involved. In the case of competition, the ionization and dissociation yields reveal the ratio between the two decay pathways. In our experiment, two observations are connected to absolute decay rates. Lifetime broadening $(<50 \mathrm{ps})$ may reveal very shortlived states in the wavelength spectra. Very long-lived states (lifetimes $>20 \mathrm{~ns}$ ) will produce distorted kinetic energy release features in our coincidence spectrum.

By scanning the laser over $5 \mathrm{~nm}(343.3-348.3 \mathrm{~nm})$ we induce transitions from the initial $c^{3} \Pi_{u}^{-}$state $(v=0-4)$ to high Rydberg states, which subsequently predissociate to the first excited dissociation limit. A sample of the raw data is presented in Fig. 3(a). The data are integrated over thousands of laser shots; in the middle of the picture one can see the shadow of the flag used to block the undissociated parent beam. Each dissociation event consists of two fragments detected in coincidence and having the center of mass confined 


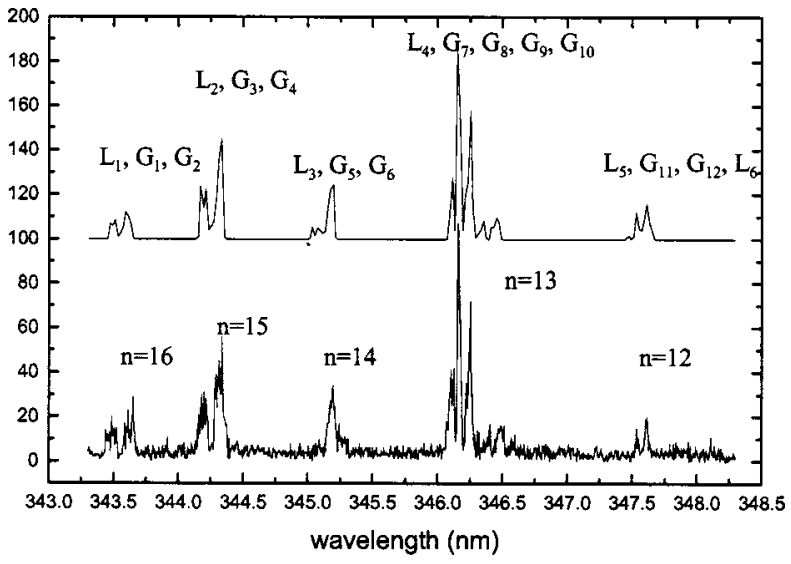

FIG. 4. Predissociation scan of $v=0$ Rydberg states. In the upper part the fit of the predissociation scan is shown. The observed lines are indicated. Often few rotational transitions cannot be resolved and they appear as $G_{i}$ lines (see Table I).

in a central region of $50 \times 80$ pixels (the CCD chip has 640 $\times 480$ pixels). For each dissociation event the camera records the $(x, y)$ position of the particles as well as the arrival time $t$. Figure 3(b) shows the calculated KER spectrum [see Eq. (3)], integrated over the whole wavelength spectrum. The peaks are labeled with the initial vibrational state. The change in photon energy during the scan is much smaller than the vibrational separation. The width of the vibrational peaks is caused by the change in photon energy (maximum $50 \mathrm{meV}$ over the full scan) and the presence of different rotational states in the fast metastable beam. The spectrum also contains background signal related to spontaneous dissociative decay of vibrational excited states in the fast beam. We can distinguish between a background dissociation and a laser induced one using time selection. Photofragments arrive at the detector only in a narrow time window ( $\sim 30 \mathrm{~ns})$ related to the firing of the laser. The fragments resulting from background dissociations appear continuously distributed over the detection gate ( $\sim 200 \mathrm{~ns})$. By imposing the restriction on the arrival time of the fragments we decrease the background level by $85 \%$.

The observed KER values can be used to generate stateselective predissociation spectra. In the case of autoionization, only the molecular ion is detected, which makes it impossible to determine the initial state for each event. The predissociation process allows the investigation of the $v=0$ Rydberg levels. These states lie below the ground state of the ion $\mathrm{H}_{2}^{+}\left({ }^{2} \Sigma_{g}^{+}\right)$, therefore they appear only in the predissociation scans.

\section{A. Predissociation of $\boldsymbol{v}=\mathbf{0}$ Rydberg states}

The KER spectrum is used for selecting only predissociation events originating from $v=0$ levels (by selecting only events that have a KER between $600 \mathrm{meV}$ and 800 meV). In Fig. 4 we present the $v=0$ spectrum over the $343.3-348.3 \mathrm{~nm}$ wavelength region. Five groups of lines can be seen, corresponding to five different Rydberg states ( $n$ $=12$ to $n=16$ ). Each group contains contributions from different rotational transitions. With the exception of the $n$ $=13$ states, for each electronic Rydberg state we observe
TABLE I. Summary of $\mathrm{H}_{2}^{*}(R, N) \leftarrow \mathrm{H}_{2}\left(c^{3} \Pi_{u}^{-}\right)\left(N_{c}\right) v=0$ transitions.

\begin{tabular}{ccccc}
\hline \hline $\begin{array}{c}\text { Observed } \\
\text { lines }\end{array}$ & $\begin{array}{c}\text { Transitions } \\
\left(R, N, N_{c}\right)\end{array}$ & $\begin{array}{c}\text { Fit } \\
\text { coefficients } \\
(\%)\end{array}$ & $\begin{array}{c}\text { Rydberg } \\
\text { series }\end{array}$ & $\begin{array}{c}\text { Fit } \\
\text { coefficients } \\
(\%)\end{array}$ \\
\hline$L_{1}$ & 121 & $1.1 \pm 1.0$ & $n=16$ & 6.1 \\
$G_{1}$ & $111+232+222+212$ & $0.9 \pm 1.0$ & & \\
$G_{2}$ & $343+333+323$ & $4.1 \pm 0.5$ & & \\
& 121 & $4.8 \pm 0.5$ & $n=15$ & 20.8 \\
$L_{2}$ & $111+232+333+212$ & $3.8 \pm 1.0$ & & \\
$G_{3}$ & $343+333+323$ & $12.2 \pm 2.0$ & & \\
$G_{4}$ & 121 & $0.6 \pm 1.0$ & $n=14$ & \\
$L_{3}$ & $111+232+222+212$ & $0.7 \pm 1.0$ & & \\
$G_{5}$ & $343+333+323$ & $9.2 \pm 1.0$ & & \\
$G_{6}$ & 121 & $6.4 \pm 1.0$ & $n=13$ & \\
$L_{4}$ & $232+111+222$ & $21.6 \pm 2.0$ & & \\
$G_{7}$ & $212+343+333+323$ & $19.6 \pm 2.0$ & & \\
$G_{8}$ & $454+444+434$ & $1.9 \pm 1.0$ & & \\
$G_{9}$ & $555+565+545$ & $3.4 \pm 0.5$ & & \\
$G_{10}$ & 121 & $0.3 \pm 0.5$ & $n=12$ & \\
$L_{5}$ & $232+222+111$ & $2.5 \pm 0.5$ & & \\
$G_{11}$ & $212+343+333$ & $3.9 \pm 0.5$ & & \\
$G_{12}$ & 323 & $2.8 \pm 1.0$ & & \\
$L_{6}$ & & & \\
\hline \hline
\end{tabular}

${ }^{\mathrm{a}} L_{i}$ labels single transitions; $G_{i}$ labels a group of transitions.

${ }^{\mathrm{b}}$ Contributing rotational transitions are indexed after quantum numbers $R, N$, and $N_{c}$.

${ }^{\mathrm{c}}$ Integrated predissociation yield for one Rydberg state.

contributions of eight rotational transitions, originating from $c^{3} \Pi_{u}^{-}\left(N_{c}=1-3\right)$ via Rydberg states characterized by $R$ $=N_{c}=1-3$ and $N=1-4$. The $n=13$ state is the strongest and 15 rotational transitions contribute to the spectrum. The transitions are characterized by $R=N_{c}=1-5$ and $N=1-6$.

In the upper part of Fig. 4 we present a least squares linear fit of the $v=0$ predissociation spectrum. We use the calculated energies of 46 transitions that are expected to appear in the (343.3-348.3 nm) wavelength region to generate 46 Gaussian distributions representing the individual rotational transitions. The resolution of our experiment does not allow us to resolve the rotational spectrum completely. Out of the 46 transitions we identified six individual rotational transition $L_{1}-L_{6}$ and 12 groups of transitions $G_{1}-G_{12}$. These distributions appearing at wavelengths fixed by the $a b$ initio calculation are used for the fitting of the predissociation spectrum. The fit is a linear combination of 18 lines:

$$
y(\lambda)=\sum a_{l} L_{l}\left(R, N, N_{c}, \lambda\right)+\sum b_{l} G_{l}(\lambda) .
$$

The $L_{l}$ refer to individual rotational transitions indexed by the quantum numbers $R, N$, and $N_{c}$, while $G_{l}(\lambda)$ $=\sum L\left(R, N, N_{c}, \lambda\right)$ describe transitions that are not resolved. The results, consisting of fit coefficients $a_{l}$ and $b_{l}$, are presented in Table I. These 18 coefficients describe the predissociation yield of the 18 lines. The unresolved rotational transitions that contribute to each $G_{l}$ line are specified by their index $\left(R, N, N_{c}\right)$.

As mentioned earlier, the intensity of the peaks represents the excitation probability in combination with the population of the initial state. Only in the case of two or 
TABLE II. Details of the $\mathrm{H}_{2}^{*}(n=13, R, N) \leftarrow \mathrm{H}_{2}\left(c^{3} \Pi_{u}^{-}\right)\left(N_{c}\right) v=0$ transitions.

\begin{tabular}{|c|c|c|c|c|c|c|}
\hline \multicolumn{4}{|c|}{$A b$ initio } & \multirow[b]{2}{*}{$\begin{array}{c}\text { Observed } \\
\text { lines }\end{array}$} & \multirow{2}{*}{$\begin{array}{c}\text { Fit } \\
\text { coefficients } \\
(\%)\end{array}$} & \multirow{2}{*}{$\begin{array}{l}\text { KER analysis } \\
\text { coefficients } \\
(\%)\end{array}$} \\
\hline $\begin{array}{l}\text { Transitions } \\
\left(R, N, N_{c}\right)\end{array}$ & $\begin{array}{l}\text { Energy }{ }^{\mathrm{a}} \\
\left(\mathrm{cm}^{-1}\right)\end{array}$ & $\begin{array}{c}\text { Transitions } \\
\left(\mathrm{cm}^{-1}\right)\end{array}$ & $\begin{array}{l}\text { KER } \\
(\mathrm{meV})\end{array}$ & & & \\
\hline 121 & 123827.3 & 28885.58 & 676.0 & $L_{4}$ & 12.2 & 12.2 \\
\hline 232 & 123943.7 & 28881.18 & 690.4 & $G_{7}$ & 40.9 & $11.5^{\mathrm{c}}$ \\
\hline 111 & 123821.9 & 28880.20 & 675.3 & & & 17.9 \\
\hline 222 & 123942.3 & 28879.80 & 690.3 & & & $11.5^{\mathrm{c}}$ \\
\hline 212 & 123939.6 & 28877.04 & 689.9 & $G_{8}$ & 36.9 & 4.5 \\
\hline 343 & 124117.7 & 28875.17 & 712.0 & & & $10.8^{\mathrm{c}}$ \\
\hline 333 & 124117.1 & 28874.65 & 711.9 & & & $10.8^{\mathrm{c}}$ \\
\hline 323 & 124114.9 & 28872.44 & 711.7 & & & $10.8^{\mathrm{c}}$ \\
\hline 454 & 124347.8 & 28867.46 & 740.5 & $G_{g}$ & 3.6 & $1.2^{\mathrm{c}}$ \\
\hline 444 & 124347.7 & 28867.34 & 740.5 & & & $1.2^{\mathrm{c}}$ \\
\hline 434 & 124345.7 & 28865.39 & 740.3 & & & $1.2^{\mathrm{c}}$ \\
\hline 555 & 124632.5 & 28858.05 & 775.8 & $G_{10}$ & 6.6 & $2.2^{\mathrm{c}}$ \\
\hline 565 & 124632.4 & 28857.95 & 775.8 & & & $2.2^{\mathrm{c}}$ \\
\hline 545 & 124630.7 & 28856.28 & 775.6 & & & $2.2^{\mathrm{c}}$ \\
\hline
\end{tabular}

${ }^{\mathrm{a}} \mathrm{Ab}$ initio calculation using Eyler and Pipkin formalism for the energy of rotationally excited Rydberg states.

${ }^{\mathrm{b}}$ From KER analysis the contribution of different $N_{c}$ states is estimated.

${ }^{c}$ These transitions initiated from the same $N_{c}$ state cannot be resolved and they appear as with equal contribution.

more decay channels is the intensity also determined by the relative decay rate of the different channels. The spectrum in Fig. 4 shows a surprising odd-even oscillation in intensity; the $n=13$ and $n=15$ are clearly more intense than the neighboring states. The fit coefficients reveal this very clearly (see Table I). Table I also shows that the contribution of the pararotational levels $\left(N_{c}=2\right)$ is weaker than the contribution of ortho-rotational levels (odd $N_{c}$ ). The $n=13$ Rydberg state is particularly strong. Possible reasons for an enhanced excitation probability are discussed below.

The $a b$ initio model developed by Eyler and Pipkin ${ }^{11}$ describes the absolute positions of the $v=0$ levels very well. Only a small shift was needed to improve the agreement. This small shift implies an overall correction of the quantum defect of $-0.01 \pm 0.005$. The values of the quantum defects, using Eq. (4), vary from 0.002 for $n=12, R=1, N=2$ Rydberg state to 0.055 for $n=16, R=1, N=1$ Rydberg state, indicating that the $d$-Rydberg states are degenerate with the high $l$ series.

The $n=13, v=0$ Rydberg states show a particularly strong predissociation behavior. The strong yield makes it possible to further distinguish the individual transitions by selecting within the $v=0$ KER peak also the different rotational levels. Table II shows these results together with the calculated $n=13, v=0$ rotational transitions that are taken into account in the fit. The ab initio formalism of Eyler and Pipkin has been used to calculate the energy of the Rydberg rotationally excited states. The photon energies as well as the KER corresponding to each of these transitions are shown. One can see that some of the rotational transitions appear almost at the same excitation frequencies (i.e., 232, 111, and 222 forming the $G_{7}$ line). The frequency difference for these transitions is less than $1 \mathrm{~cm}^{-1}$, a splitting that our experimental resolution cannot resolve. Still, transitions that originate from different rotational states $\left(N_{c}=1\right.$ and $\left.N_{c}=2\right)$ have different kinetic energy release values (see Table II).

We separate the rotational transitions by analyzing only events with KER of $660-680 \mathrm{meV}$. This spectrum has a dominant contribution from $N_{c}=1$ transitions. We repeat the selection of dissociation events for KER values between 680 and $700 \mathrm{meV}$, corresponding to $N_{c}=2$ transition as well as for $700-725 \mathrm{meV}$, corresponding to $N_{c}=3$ transition. Three spectra have been obtained, which contain dominantly rotational transitions starting from one defined $N_{c}$ state. By fitting these three spectra with $N_{c}=1, N_{c}=2$, and $N_{c}=3$ transitions, respectively, we obtain the relative contributions of different rotational states to the $G_{7}$ and $G_{8}$ lines of $v=0$ spectrum. We stress here that this method helps us to estimate the contribution of different rotational transitions to the same line, but it does not resolve the contribution of different transitions starting from the same rotational state $N_{c}$ (i.e., 232 and 222 forming the $G_{7}$ line). These transitions cannot be resolved and therefore they appear in Table II with equal coefficients.

Despite the limited spectral resolution, the use of the KER analysis makes it possible to quantify the contribution of the different initial rotational states to the observed intensity. This is certainly relevant as an indication for the nature of the perturbations that affect the observed peak strengths. Figure 5 compares the observed contributions of the different rotational states with an expected population using a thermal rotational distribution at $P\left(N_{c}\right)=\left(2 N_{c}\right.$ $+1) c e^{\left[-\beta_{e} N_{c}\left(N_{c}+1\right)\right] / k T}$ at $T=350 \mathrm{~K}$. Under inclusion of the para-ortho modulation $c$, we observe a satisfactorily agreement. We conclude that the large intensity of the $n=13$ Rydberg state is not a consequence of a rotational state dependent interaction with another state.

As we have described before, these experiments have been performed in the presence of a weak electric field of about $200 \mathrm{~V} \mathrm{~cm}^{-1}$ (see Fig. 2). The effect of this small field differs for the $v=0$ levels in comparison to $v=1$ levels. Whereas for the $v=1$ levels, the observed predissociation yield changes significantly, this is not the case for $v=0 \mathrm{lev}$ els. In Fig. 6 we present two scans over the $G_{7}$ line $n=13$, 


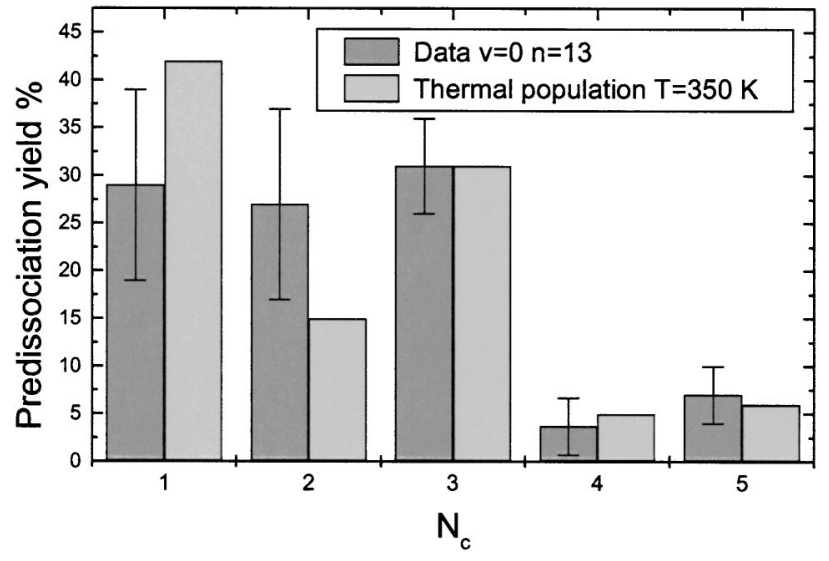

FIG. 5. Comparison between the rotational $\left(R=N_{c}\right)$ contribution to the $n=13, v=0$ spectrum and an expected rotational population of the $c^{3} \Pi_{u}^{-}(v=0)$ at $T=350 \mathrm{~K}$.

$v=0$, with and without the electric field. No difference is observed in the predissociation yield. A change in width is observed and it is attributed to the Stark structure of the states involved. The resolution of the experimental setup $\left(\sim 0.2 \mathrm{~cm}^{-1}\right.$ laser bandwidth and $\sim 0.2 \mathrm{~cm}^{-1}$ Doppler shift) in combination with the fact that $G_{7}$ line contains contribu-
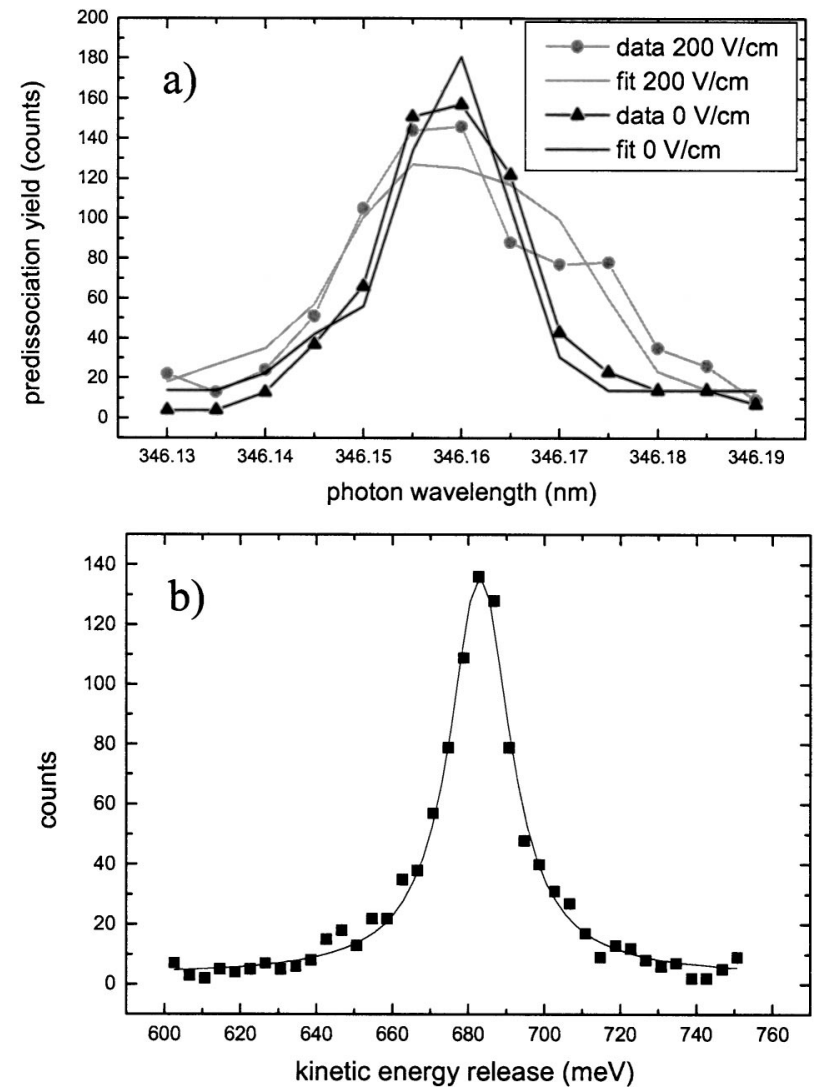

FIG. 6. (a) Two scans over $G_{7}$ line $(n=13, v=0)$ in the absence of the electric field and at $200 \mathrm{~V} / \mathrm{cm}$ electric field. The width of the experimental lines can be fitted by taking into account the Doppler broadening $\left(0.2 \mathrm{~cm}^{-1}\right)$, the laser bandwidth $\left(0.2 \mathrm{~cm}^{-1}\right)$, and the Stark splitting. No indication of lifetime broadening is present suggesting lifetimes longer than 50 ps. (b) The KER histogram over $G_{7}$ line in the absence of electric field shows no significant asymmetry. A lifetime longer than $10 \mathrm{~ns}$ would appear as a tale at lower KER values. tions from three transition 232, 111, and 222, separated by $\sim 1$ and $\sim 0.4 \mathrm{~cm}^{-1}$, respectively, accounts for the width of the line in the absence of electric field [see Fig. 6(a)]. In the presence of electric field, the $n$-fold degeneracy of each rotational Rydberg states is lifted and the Rydberg state splits into a Stark manifold of $k$ states. The energy of each Stark state can be approximated by $E=-1 / 2 n^{2}+3 / 2 F n k$, where the quantum number $k$ runs from $(n-1, n-3, \ldots,-n+1)$ for $m=0$ and $(n-2, n-4, \ldots,-n+2)$ for $m=1$ and $F$ is the electric field (a.u.). ${ }^{32}$ The splitting between two consecutive Stark states of $n=13$ Rydberg state, in the presence of a 200 $\mathrm{V} \mathrm{cm}{ }^{-1}$ electric field, is $\sim 0.166 \mathrm{~cm}^{-1}$. The maximum split (for $k=n-1,-n+1$ ) is $\sim 4 \mathrm{~cm}^{-1}$. Taking into account the Stark splitting, we can reproduce the width of the $G_{7}$ line [see Fig. 6(a)].

As mentioned before, the experiment makes it possible to deduce a lifetime of the upper state only if the lifetime broadening can be observed in the spectrum or, in case of very long lifetimes, from the tail of the KER peaks at smaller apparent KER values. From the analysis of the KER distributions we do not observe any asymmetry [see Fig. 6(b)]. No clear lifetime effect has been observed, neither with nor without the electric field. In both cases, the natural linewidth is smaller than $0.5 \mathrm{~cm}^{-1}$. The above observations do not imply that the electric field has no effect on the predissociation lifetime. If the lifetime is reduced or enhanced in the range from $50 \mathrm{ps}$ to $10 \mathrm{~ns}$, this would not result in a change in the observed quantities. We have only shown that predissociation is the dominant decay channel for these Rydberg levels in the presence and in the absence of a static field. As will be shown below, in the case of the $v=1$ levels, the predissociation yield depends strongly on the external electric field.

\section{B. Predissociation and autoionization of $v=1-4$ Rydberg states}

For excited vibrational Rydberg states, autoionization becomes an open decay channel competitive to the predissociation channel. Since the lowest triplet unbound $Q^{3} \Pi_{g}$ state, leading to the $n=2$ dissociation limit crosses the Rydberg states around $v=5-6$ (Refs. 17, 18, and 24), direct predissociation of $v=1$ Rydberg states is very unlikely. In contrast to this, in the presence of an electric field, we observed predissociation signal from $v=1, n=13-18$ Rydberg states [see Fig. 3(b)] as well as from $v=2-4$ Rydberg states.

By selecting events having a KER between $950 \mathrm{meV}$ and $1050 \mathrm{meV}$ we select transitions initiated from $c^{3} \Pi_{u}^{-}(v$ $=1$ ) rotational states. The predissociation spectrum is presented in the lower part of Fig. 7. With photon wavelengths between $343.3 \mathrm{~nm}$ and $348.3 \mathrm{~nm}$ we excite the $n=13-20$ Rydberg states $(v=1)$. The quality of the data does not permit a quantitative measurement of the predissociation yield for independent rotational transitions $\left(R, N, N_{c}\right)$. Most of the peaks in the predissociation spectrum can be assigned. For clarity in Fig. 7 we show the position of the 111 transitions. The predissociation spectra of Rydberg series $(v=2-4)$ are obtained in similar way using KER restrictions. The spectra are presented in Fig. 7 together with the position of the 111 transitions of each $n$-Rydberg state. The lowest $n$-Rydberg 


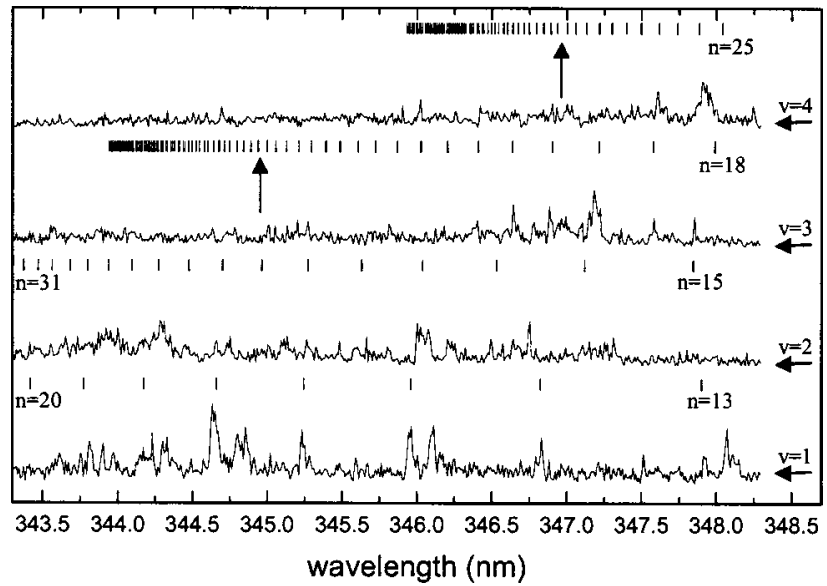

FIG. 7. Predissociation scans of $v=1-4$ Rydberg states. For clarity only the 111 transitions are shown (field free). The two arrows show the value of the IP in the presence of a $200 \mathrm{~V} \mathrm{~cm}^{-1}$ electric field.

state excited is specified for each vibrational spectrum. For $v=3$ and $v=4$ Rydberg series all the $n$-Rydberg states with $n>18$ and $n>25$, respectively, are excited.

The scans have been performed in the presence of a 200 $\mathrm{V} \mathrm{cm}^{-1}$ electric field in the laser interaction region. The Stark splitting of the Rydberg states increases with $n$ and the maximum Stark splitting increases with $n(n-1)$. At high values of $n$, the different rotational transitions overlap due to Stark splitting. For $v=4$ Rydberg states, the transition energy difference between different rotational states is larger than the $n$ spacing. As a consequence, the rotational transitions of different Rydberg states mix and appear less as individual lines and more as a continuous band. The same continuous band appearance can be seen for the $v=3, n$ $>23$ Rydberg states. Another effect of the electric field is the occurrence of a saddle point in the potential energy surface in the direction of the field. The ionization potential (IP) is lowered with $\Delta E=-\alpha \sqrt{F}$, where $\alpha=6.12 \mathrm{~cm}^{-1}$ and $F$ is the electric field expressed in $\mathrm{V} \mathrm{cm}^{-1}$. For a $200 \mathrm{~V} \mathrm{~cm}^{-1}$ electric field, the IP is lowered by $\sim 86.5 \mathrm{~cm}^{-1}$. The Rydberg states characterized by $n<35$ are situated below this saddle point. The Rydberg series with $n>35$ are above the saddle point and they ionize directly. The two arrows in Fig. 7 indicate the positions of the IP values of the $v=3$ and $v=4$ levels in a $200 \mathrm{~V} \mathrm{~cm}^{-1}$ field.

The $v=1$ Rydberg states are energetically above the $\mathrm{H}_{2}^{+}\left({ }^{2} \Sigma_{g}^{+}\right)(v=0)$ ionic state and autoionization is possible. Autoionization is faster than predissociation. At zero electric field autoionization is the dominant decay channel for the $v$ $=1$ Rydberg states and predissociation is zero. The predissociation channel opens in the presence of the field (already at $25 \mathrm{~V} \mathrm{~cm}^{-1}$ ) and it shows a fast increase with the electric field. In Fig. 8(a) we present the predissociation yield of $n$ $=15, v=1, R=1$ Rydberg state versus the electric field. At zero electric field the predissociation yield is zero (background level). By increasing the electric field up to 100 $\mathrm{V} \mathrm{cm}{ }^{-1}$ the predissociation yield increases fast. Between 100 and $200 \mathrm{~V} \mathrm{~cm}^{-1}$ the predissociation yield remains constant within the experimental error. In Fig. 8(b) we present the autoionization yield versus electric field. The autoionization
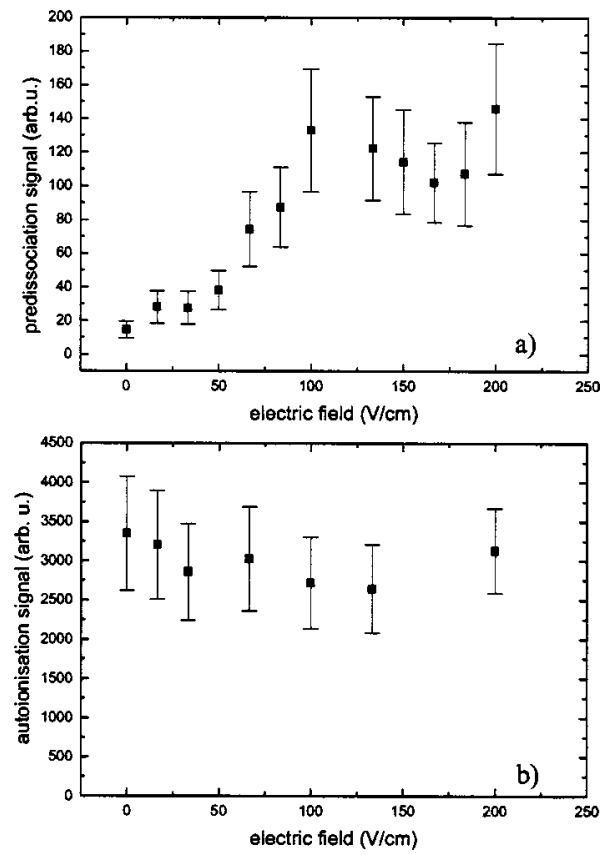

FIG. 8. (a) The predissociation yield of $n=15, v=1, R=1$ Rydberg states versus electric field. (b) The autoionization yield of the same Rydberg states versus electric field.

signal shows no significant dependence on the electric field. For electric fields higher than $100 \mathrm{~V} \mathrm{~cm}^{-1}$, the predissociation yield accounts for $8 \%$ of the decay of the $n=15, v$ $=1, R=1$ Rydberg states, the rest $92 \%$ decaying via autoionization. We conclude that the electric field does not significantly affect the excitation probability, represented by the sum of autoionization and predissociation signal, but it does affect the relative rates of these two processes.

The absence of predissociation signal for $v=1$ Rydberg states at zero electric field indicates that the $d$ character of the Rydberg electron renders the predissociation a rate sufficiently small such that the competition with autoionization is lost. In general, the autoionization as well as predissociation depends on the angular momentum of the outer electron. Both interactions require a collision of the Rydberg electron with the ionic core. This collision becomes very ineffective for high angular momentum states $(l>3)$. The effect of the electric field on the predissociation fraction suggests strongly that the $p$ and $s$ character, which the electrons acquire in the static electric field, drives the predissociation process. It is of interest to note here that the angular momentum mixing in Hund's case $d$ implies a mixing of gerade and ungerade character.

An ionization spectrum was obtained by counting the deflected laser induced ions onto the detector (see the gray spectrum in Fig. 9). The ionization spectrum contains much more lines than the predissociation spectrum mainly because the ionization spectrum has contributions from all vibrational states that are excited, while predissociation spectrum shows only $v=1$ transitions. In order to measure the branching ratios between the predissociation and autoionization, we set the laser wavelength on the most dominant predissociation transitions, usually characterized by $R=N_{c}=1$ and $R=N_{c}$ 


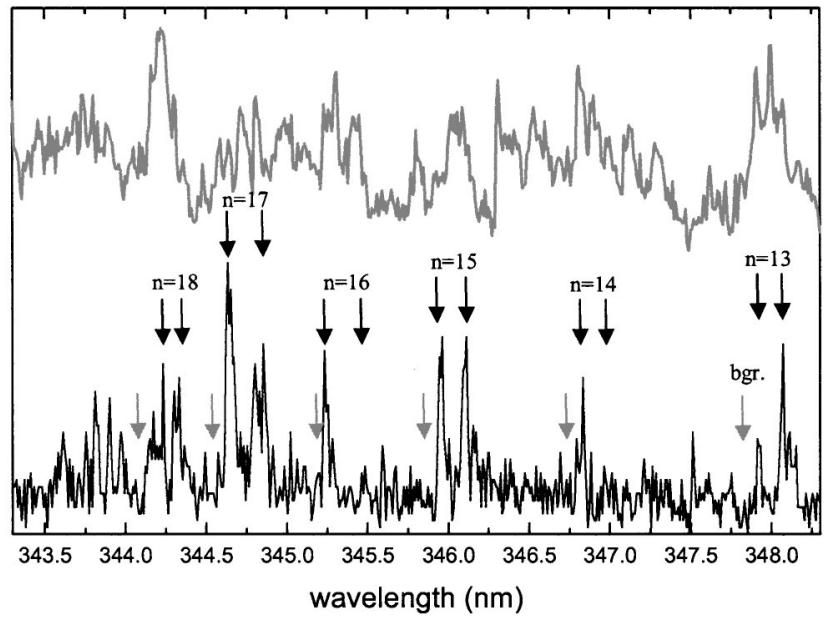

FIG. 9. Predissociation scan of $v=1$ Rydberg states (black spectrum) versus autoionization scan (gray spectrum). The branching ratios are measured at 10 photon wavelengths corresponding to $R=N_{c}=1$ and $R=N_{c}=3$ transitions. The background is measured at photon wavelengths indicated by the gray arrows.

$=3$. Both predissociation and autoionization signals are corrected for background.

The photon wavelengths corresponding to background measurements are chosen in the vicinity of the $v=1$ transitions, as it is shown by the arrows in Fig. 9. In case of predis-sociation, after selection of the laser induced events, the background yield is low and the predissociation is accurately measured. In case of autoionization, due to the complexity of the spectrum, the background yield is much less accurately measured. It is still possible to estimate the competition between the two decay channels.

In case of odd and even principal quantum numbers, the average predissociation rate is $\sim 7 \%$ and $\sim 2 \%$, respectively.

\section{DISCUSSION AND CONCLUSIONS}

As described in the Introduction, the dissociation and autoionization dynamics of high lying Rydberg states are related to the relative DR rates of the different electron partial waves and total electronic multiplicity (total spin, $\mathbf{S}=0$, or $\mathbf{S}=1$ ). This connection between DR and Rydberg state dynamics should not be confused with the so-called indirect channel in $\mathrm{DR},{ }^{33}$ in which the electron-ion collision cross section shows sharp resonance features, which are attributed to the temporarily formation of quasibound, vibrationally excited, Rydberg states. In fact, these resonances in the DR cross sections in many cases reduce the cross section related to the direct DR process. ${ }^{34}$ The connection between DR and the dynamics of excited Rydberg states is inspired by the ideas behind MQDT, in which the interaction between the outer diffuse Rydberg electron is closely related to the dynamics of a low energy electron collision with the molecular ion. The advantage of studying Rydberg state dynamics is that the effect of the different electron partial waves can be isolated in principle.

This study addresses via the study of the associated Rydberg states, triplet electron collisions along the $d(\lambda)_{g}$ partial waves, and due to the electric field, also the $p(\lambda)_{u}$ and $s(\sigma)_{g}$ partial waves. In our study these Rydberg states are formed through excitation of the $2 p \pi_{u}$ electron of the metastable $c$ state. We have observed that the $d$ series are subject to predissociation in the case that autoionization is energetically not allowed. Hence this holds for the $n d_{g}, v=0$ levels. In field-free conditions, the excited vibrational levels do not show any measurable predissociation. Recent theoretical work by Florescu et al. addressing the role of triplet $\mathrm{H}_{2}$ in low energy dissociative recombination reveals a significant radial coupling strength between the electron-molecular ion channel and the $i^{3} \Pi_{g}$ state. ${ }^{35}$ This state has a $3 d \pi_{g}$ as outer electron. Its potential curve shows a suitable potential barrier offering a dissociation continuum.

It is well known from the singlet system that the $n d\left(N^{+}=0\right)$ are mixed with the $n s\left(N^{+}=2\right)$ Rydberg series. This coupling reflects the approximate conserved nature of the ionic core rotational quantum number $N^{+}$in the Hund's case- $d$ description. An external electric field is causing Stark structure of the $l$-uncoupled electrons, resulting in further mixing of $s$ - and $d$ character and admixture of higher electron angular momentum states as well as $p$ character. We note that this implies that in the presence of an electric field, the Rydberg states can mix and be predissociated by ungerade lower lying states. In the present experiments, we do not find a direct evidence for the enhancement of the predissociation rate of the $v=0$ Rydberg series due to electric field. However, we find that the predissociation rate of the $v=1$ Rydberg states is enhanced by the electric field. Hence, we conclude that the $n p_{u}$ and $n s_{g}$ character can interact with a lower lying Rydberg state offering a dissociative continuum. Florescu and co-workers have evaluated the radial coupling strength of the continuum low energy electrons with the different open dissociation channels and they have found that the $\left(3 s \sigma_{g}\right) h^{3} \Sigma_{g}^{+}$state has the strongest interaction. The strongest ungerade channel is the $\left(3 p \sigma_{u}\right) e^{3} \Sigma_{u}^{+}$state, a state with a quantum defect that is unusually large for molecular hydrogen. According to Florescu et al., the importance of this state in the DR cross section is in between that of the $h^{3} \Sigma_{g}^{+}$and the $i^{3} \Pi_{g}$ states. We conclude that our observations are in agreement with the $h$ state to be the dominant channel.

The effect of the electric field on the predissociation rate of $v=1$ levels is one of the most interesting results of the present work. The electric field has two potential effects. First, it may cause the $l=2$ character of the Rydberg electron to become nonconserved, resulting in a transition to $k$ quantum states. Second, it may affect the long-range interaction causing the splitting as described by Eq. (4). With regard to the latter we note that at a field of $200 \mathrm{~V} \mathrm{~cm}^{-1}$, the $n=13$, $v=0$ manifold of lines is still well described ignoring the presence of the electric field. The spectral details of the $v$ $=1$ lines prevent drawing a similar firm conclusion. With regard to the first effect, we note that the Stark splitting of the $l=2$ Rydberg states implies directly mixing with the higher $l$ states and after lifting the degeneracy with the $l$ $=1$ and $l=0$ Rydberg states also mixing with the $n p \lambda_{u}$ and $n s \sigma_{g}$ Rydberg levels. To assess the electric fields at which the mixing occurs, the position of the $n p \lambda_{u}$ and $n s \sigma_{g}$ Rydberg levels is important. Whereas Eq. (4) provide an accurate description of the $L>2$ states, this description is inaccurate 
for the $L=0,1$ states. Fortunately the quantum defects of the $4 s^{3} \Sigma_{g}^{+}$state is known to be $0.03 .{ }^{28}$ This places the $(R L N)$ $=(122)$ level about $1.7 \mathrm{~cm}^{-1}$ above the $(R L N)=(101)$ level, whereas the $(R L N)=(121)$ is about $2.5 \mathrm{~cm}^{-1}$ below the $(R L N)=(101)$ level. A field of about $100 \mathrm{~V} \mathrm{~cm}^{-1}$ provides a width of the manifold of $n=15, v=1$ states that causes the $L=0$ Rydberg state to be degenerate. This observation provides support for the hypothesis that mixing with the $15 s(\sigma)_{g}$ Rydberg state provides the doorway to the predissociation channel for the $L=2$ Rydberg states.

This triplet $3 d$ channel in molecular hydrogen has similarities and important differences with the singlet analog states. The difference is due to the presence of the doubly excited $\left(2 p \sigma_{u}\right)^{2}{ }^{1} \Sigma_{g}^{+}$state. Rottke and Welge have studied the $3 d$-Rydberg series using the $B^{1} \Sigma_{u}^{+}$state as intermediate state. Strong predissociation is reported but because of experimental reasons, predissociation and autoionization could not easily be distinguished in their work. These authors stress the importance of the $\left(2 p \sigma_{u}\right)^{2}$ character in the $F$ state of molecular hydrogen, implying the importance of the doubly excited curve in the ionization continuum. It is this singlet channel that carries most of the strength of the DR process with low energy electrons, ${ }^{22,36}$ in which the singlet $3 d \sigma$-partial wave dominates the DR process.

In the singlet as well as in the triplet manifolds, nearly none of the members of the $(3 s \sigma)$ Rydberg series have been observed. Their small quantum defects cause overlap with the $3 d$ series, while their oscillator strength to the $3 d$ series is stronger than the $3 s$ series by a factor of 30 . In the experiments from Rottke and Welge a small number of high lying $n s$-Rydberg states has been assigned. ${ }^{8}$ In the triplet manifold, the autoionizing experiments did not reveal any $n s$ series. Eyler and Pipkin have reported the lower lying $(4 s)^{3} \Sigma_{g}^{+}$ state, which was found to be efficiently predissociated for the levels $(v>2)$ that are situated above the barrier in the $(3 s) h^{3} \Sigma_{g}^{+}$state. ${ }^{28}$ The predissociation lifetimes are reported to be of the order of $500 \mathrm{ps}$. Using an $n^{3}$ scaling, this would give rise to predissociation lifetimes of the order of $10 \mathrm{~ns}$ for $n=13$; again the observed electric field induced predissociation in our experiments can be attributed to the $n d-n s$ mixing processes.

In a nice series of experiments, Glab and co-workers have studied the singlet $p$-ungerade series in a multiphoton excitation step via the $E^{1} \Sigma_{g}^{+}$level. ${ }^{37,38}$ Also in this system, in field-free conditions no predissociation was observed. In this research, the excited hydrogen fragments were detected using one-photon ionization with $532 \mathrm{~nm}$ light. Slow predissociation with lifetime much longer than 10 ns remains undetected in this experiment. After switching on an electric field, predissociation using excitation of the $p$-state resonances is observed, even at very small fields of $50 \mathrm{~V} \mathrm{~cm}^{-1}$. These authors point at the admixture of $n s$ character to explain the predissociation. ${ }^{37}$ In a second paper by Glab and Qin the competition between autoionization and dissociation is observed starting from the same Rydberg states with an excited $v=1$ core ${ }^{38}$ Here direct evidence is given for the $n d$ states to be responsible for predissociation.

To the best of our knowledge, no reports exist on the triplet $p$-ungerade Rydberg states. Lindsay et al. have studied the $n f$ series using autoionization as probe. ${ }^{7}$ They remark on the large effects of an external electric field up to 1000 $\mathrm{V} \mathrm{cm}^{-1}$, which decreases the signal to a large extent. The accuracy of the wavelength scans in their work allowed detection of perturbations directly in linewidths. It is of interest to note that also in the $n f$-series, perturbations are found for the Rydberg states with principal quantum number $n=11$, 13, and 15. Like in this work, it has not been possible to identify the perturber state which enhances the decay of odd Rydberg states.

In conclusion, a series of reports exist on the spectroscopy of many Rydberg series in molecular hydrogen. In combination with Stark fields, most of the partial waves have been addressed experimentally. In spite of all this research, the decay mechanisms have only been touched often indirectly. Because of the importance of this decay channels for understanding dynamic processes such as dissociative recombination, it is of relevance to quantify the partial autoionization and dissociation decay widths of the different Rydberg series, triplet as well as singlet.

\section{ACKNOWLEDGMENTS}

The work described in this paper is part of the research program of the Foundation for Fundamental Research on Matter (Stichting voor Fundamenteel Onderzoek der Materie) and it was financially supported by the Netherlands Organization for Scientific Research (Nederlandse Organisatie voor Wetenschappelijk Onderzoek) through the FOM program Molecular Atmospheric Processes. The authors thank H. G. Muller, A. I. Florescu, and A. Suzor-Weiner for their comments and suggestions.

${ }^{1}$ W. Kolos and J. Rychlewski, J. Mol. Spectrosc. 66, 428 (1977).

${ }^{2}$ W. Kolos and J. Rychlewski, J. Mol. Spectrosc. 143, 237 (1990).

${ }^{3}$ L. Wolniewicz, J. Mol. Spectrosc. 169, 329 (1995).

${ }^{4}$ S. Ross and Ch. Jungen, Phys. Rev. Lett. 59, 1297 (1987).

${ }^{5}$ S. C. Ross and Ch. Jungen, Phys. Rev. A 49, 4353 (1994).

${ }^{6}$ E. E. Eyler, R. C. Short, and F. M. Pipkin, Phys. Rev. Lett. 56, 2602 (1986)

${ }^{7}$ M. D. Lindsay, A. W. Kam, J. R. Lawall, P. Zhao, F. M. Pipkin, and E. E. Eyler, Phys. Rev. A 41, 4974 (1990).

${ }^{8}$ H. Rottke and K. H. Welge, J. Chem. Phys. 97, 908 (1992).

${ }^{9}$ D. P. de Bruijn and J. Los, Rev. Sci. Instrum. 53, 1020 (1982).

${ }^{10}$ H. Helm, D. P. de Bruijn, and J. Los, Phys. Rev. Lett. 53, 1642 (1984).

${ }^{11}$ E. E. Eyler and F. M. Pipkin, Phys. Rev. A 27, 2462 (1983).

${ }^{12}$ R. D. Knight and L. Wang, Phys. Rev. Lett. 55, 1571 (1985).

${ }^{13}$ R. Kachru and H. Helm, Phys. Rev. Lett. 55, 1575 (1985).

${ }^{14}$ W. G. Sturrus, E. A. Hessels, P. W. Arcuni, and S. R. Lundeen, Phys. Rev. A 44, 3032 (1991).

${ }^{15}$ Z. W. Fu, E. A. Hessels, and S. R. Lundeen, Phys. Rev. A 46, R5313 (1992).

${ }^{16}$ A. Osterwalder, R. Seiler, and F. Merkt, J. Chem. Phys. 113, 7939 (2000).

${ }^{17}$ N. Bjerre, S. R. Keiding, L. L. Lembo, and H. Helm, Phys. Rev. Lett. 60, 2465 (1988).

${ }^{18}$ L. J. Lembo, N. Bjerre, D. L. Huestis, and H. Helm, J. Chem. Phys. 92 , 2219 (1990).

${ }^{19}$ A. Matzkin, Ch. Jungen, and S. C. Ross, Phys. Rev. A 62, 062511 (2000).

${ }^{20}$ D. R. Bates, Phys. Rev. 78, 492 (1950).

${ }^{21}$ D. Auerbach, R. Cacak, R. Caudano, T. D. Gaily, C. J. Keyser, J. M. McGowan, J. B. A. Mitchell, and S. F. J. Wilk, J. Phys. B 10, 3797 (1977).

${ }^{22}$ A. Giusti-Suzor, J. N. Bardsley, and C. Derkits, Phys. Rev. A 28, 682 (1983).

${ }^{23} \mathrm{H}$. Takagi, in Dissociative Recombination: Theory, Experiment and Application IV, edited by M. Larsson, J. B. A. Mitchell, and I. F. Schneider (World Scientific, Singapore 1999), p. 180. 
${ }^{24}$ S. L. Guberman, J. Chem. Phys. 78, 1404 (1983).

${ }^{25}$ B. K. Sarpal, J. Tennyson, and L. A. Morgan, J. Phys. B 27, 5943 (1994).

${ }^{26}$ D. Strasser, X. Urbain, H. B. Pedersen, N. Altstein, O. Heber, R. Wester, K. G. Bhushan, and D. Zajfman, Rev. Sci. Instrum. 71, 3092 (2000).

${ }^{27}$ L. Dinu, A. T. J. B. Eppink, F. Rosca-Pruna, H. L. Offerhaus, W. J. van der Zande, and M. J. J. Vrakking, Rev. Sci. Instrum. 73, 4206 (2002).

${ }^{28}$ E. E. Eyler and F. M. Pipkin, J. Chem. Phys. 77, 5315 (1982).

${ }^{29} \mathrm{H}$. M. Crosswhite, The Hydrogen Molecule Wavelength Tables of Gerhard Heinrich Dieke (Wiley, New York, 1972).

${ }^{30}$ T. A. Miller and R. S. Freund, J. Chem. Phys. 61, 2160 (1974).
${ }^{31}$ K. P. Huber and G. Herzberg, Constants of Diatomic Molecules (Van Nostrand Reinhold, New York, 1979).

${ }^{32}$ F. T. Gallagher, Rydberg Atoms (Cambridge University Press, Cambridge, 1994).

${ }^{33}$ J. N. Bardsley, J. Phys. B 1, 349 (1968).

${ }^{34}$ A. Giusti, J. Phys. B 13, 3867 (1980).

${ }^{35}$ A. I. Florescu, V. Ngassam, I. F. Schneider, and A. Suzor-Weiner, J. Phys. B 36, 1205 (2003).

${ }^{36}$ H. Takagi, J. Phys. B 26, 4815 (1993).

${ }^{37}$ K. Qin, M. Bistransin, and W. L. Glab, Phys. Rev. A 47, 4154 (1993).

${ }^{38}$ W. L. Glab and K. Qin, J. Chem. Phys. 99, 2345 (1993). 